\title{
Predicting the response of patients with advanced urothelial cancer to methotrexate, vinblastine, Adriamycin, and cisplatin (MVAC) after the failure of gemcitabine and platinum (GP)
}

\author{
Ki Hong Kim, Sung Joon Hong and Kyung Seok Han ${ }^{*}$
}

\begin{abstract}
Background: Platinum-based systemic chemotherapy is the treatment of choice for patients with advanced urothelial carcinoma (UC). Although no chemotherapeutic regimen is established as a second-line therapy, recent studies reported that methotrexate, vinblastine, Adriamycin and cisplatin (MVAC) elicited a significant response in patients who failed gemcitabine and platinum (GP) chemotherapy. We investigated the clinical factors useful for predicting a favourable response to MVAC in UC patients who failed GP.

Methods: Forty-five patients with advanced UC who received second-line MVAC chemotherapy after failure with first-line GP chemotherapy were enrolled in this study. Univariate and multivariate analyses based on Cox's regression were performed to identify independent prognostic factors for progression-free survival (PFS) after second-line MVAC chemotherapy.

Results: The median follow-up period after the first MVAC administration was 10.0 months. The median PFS and overall survival (OS) were 6.5 months (95\% confidence interval [Cl]: 5.1-7.9) and 14.5 months (95 \% Cl, 7.4-21.4), respectively. The overall response rate was $57.8 \%$. The response to first-line GP chemotherapy (hazard ratio [HR], $2.500 ; p=0.012)$ and patient age (HR, 1.047; $p=0.033)$ were predictors of PFS after MVAC chemotherapy.

Conclusions: The response to first-line GP chemotherapy and age were independent predictors of PFS in patients who received second-line MVAC chemotherapy. This report is the first to describe independent predictors of PFS after MVAC chemotherapy.
\end{abstract}

Keywords: Urothelial carcinoma, Chemotherapy, Cisplatin, Second-line

\section{Background}

Systemic chemotherapy is the treatment of choice for metastatic, recurrent or inoperable urothelial carcinoma (UC). Methotrexate, vinblastine, Adriamycin and cisplatin (MVAC) chemotherapy was used worldwide as the standard treatment since the first report of its efficacy in 1985 [1]. However, since gemcitabine and cisplatin (GC) chemotherapy showed similar efficacy as MVAC but with less toxicity in a large, randomized, multinational and multicentre phase III study of GC

\footnotetext{
*Correspondence: khan@yuhs.ac

Department of Urology and Urological Science Institute, Yonsei University College of Medicine, 50-1, Yonsei-ro, Seodaemun-gu, Seoul 120-752, South
} Korea

combination chemotherapy, [2] GC regimens are now used mostly as the initial systemic chemotherapy for UC.

GC chemotherapy has an excellent response rate in patients with advanced UC, and up to $20 \%$ of individuals have achieved a complete response of long-term, diseasefree survival [3]. However, most patients eventually experience disease progression or relapse after GC. Several regimens have been investigated in the second-line setting after the failure of GC, including taxanes, vinflunine, ifosfamide, ixabepilone and pemetrexed; [4-9] however, no regimen has yet achieved a competent survival benefit in second-line setting.

Recently, a small number of clinical trials proposed that platinum-based regimens are effective in a significant 
portion of patients in whom platinum-based chemotherapy initially failed when the regimen was based on cisplatin but using different agents in the second-line setting. The efficacy and safety of MVAC chemotherapy as a second-line treatment after the failure of gemcitabine and platinum (GP) chemotherapy has been reported in several small-scale studies [10-13]. However, there is a lack of predictive factors available for the personalized selection of chemotherapeutic regimens in patients with UC. Therefore, we investigated the factors predictive of a favourable response to second-line MVAC chemotherapy after GP in patients with advanced UC to facilitate the development of a tailored second-line treatment strategy. Here, we investigated the predictive value of the response to first-line GP chemotherapy for selecting suitable candidates for the MVAC regimen as the second-line chemotherapy in patients with advanced UC.

\section{Methods}

\section{Study population}

The medical ethics committee of Severance Hospital, Yonsei University Health Care System (Seoul, Korea) approved this retrospective study. Our medical ethics committee allows exempt of informed consent if the research uses the collection or study of existing data, documents and records and these sources are publicly available and the information is recorded by the investigator in such a manner that subjects cannot be identified. From these regulations, our study was classified as exempt from the informed consent requirement because this study was based on the collection of existing publicly available data, documents and records, and we recorded all data in a manner that subjects cannot be identified. Between July 2004 and August 2014, 64 consecutive patients who received MVAC chemotherapy as the second-line treatment due to the relapse or disease progression of UC after first-line GP chemotherapy in the only metastatic setting were included in this study. Nineteen of the 64 patients were excluded because of incomplete medical records (seven patients), another synchronous metastatic malignancy (five patients), an atypical carcinoma (four patients) or a history of neoadjuvant chemotherapy (three patients). Forty-five patients were included in the final analysis.

\section{Treatment}

Before starting chemotherapy, all patients were required to have an Eastern Cooperative Oncology Group (ECOG) performance status $(\mathrm{PS}) \leq 2$, adequate hematologic parameters (absolute granulocyte count $\geq 1,500 / \mathrm{dL}$, haemoglobin $\geq 8.5 \mathrm{~g} / \mathrm{dL}$ and a platelet count $\geq 100,000 / \mathrm{dL}$ ) and sufficient hepatic (serum bilirubin $\leq 1.5 \mathrm{mg} / \mathrm{dL}$ ) and renal (estimated glomerular filtration rate $\geq 50 \mathrm{~mL} / \mathrm{min}$ ) function. Methotrexate was given at a dose of $30 \mathrm{mg} / \mathrm{m}^{2}$ on days 1 ,
15 and 22, vinblastin was given at a dose of $3 \mathrm{mg} / \mathrm{m}^{2}$ on days 2, 15 and 22, Adriamycin was given at a dose of $30 \mathrm{mg} / \mathrm{m}^{2}$ on day 2 and cisplatin was given at a dose of $70 \mathrm{mg} / \mathrm{m}^{2}$ on day 2 . The cycles were repeated every 28 days, and treatment continued until the disease regressed or the toxicity was intolerable.

\section{Response and toxicity assessment and dose modification}

Basically, after the completion of three cycles, imaging tools (computed tomography scans, radionuclide bone scans or positron emission tomography) were used to evaluate the treatment response after three cycles, except for cases with symptomatic progression. Performance status, haematological parameters and liver and kidney function were measured weekly in each patient to assess for potential toxicity. The response was evaluated according to the Response Evaluation Criteria for Solid Tumours, [14] and toxicity was determined using the National Cancer Institute Common Toxicity Criteria (ver. 4.0). The platinum dose was reduced by $20-30 \%$ in patients who experienced grade 4 haematological toxicity or grade $3 / 4$ non-haematological toxicity.

\section{Outcomes}

The end point of the study was PFS in patients who received MVAC chemotherapy. The PFS of patients who received MVAC chemotherapy was defined as the time from the date of the beginning of the first MVAC cycle to the date that progression was identified, death, or loss to follow-up. Progression was defined as $a \geq 20 \%$ increase in the overall sum of the diameter of the target lesions on radiological assessments.

\section{Clinical data and statistical analysis}

All included patients were divided into good or poor response groups based on the response to the first-line GP chemotherapy. The good response group included the complete response $(\mathrm{CR})$ and partial response (PR) groups to first-line GP chemotherapy, while the poor response group included the stable disease (SD) and progression disease (PD) groups [15]. The baseline characteristics of the two groups were then compared using Chi-squared tests. PFS after MVAC chemotherapy was calculated using the Kaplan-Meier method, and statistical significance was determined using log rank tests. Age, haematological parameters, liver and kidney function, serum albumin concentration at the beginning of the first MVAC and time to progression (TTP) after first-line GP chemotherapy were included in the analysis as continuous variables. Clinical nodal status, distant metastatic status, ECOG PS at the beginning of the first MVAC and response to previous GP chemotherapy were analysed as categorical variables. Statistical analyses to identify independent predictors 
of progression after MVAC chemotherapy were performed using univariate and multivariate Cox's proportional hazard regression analyses. Variables that were significant in the univariate analysis $(p<0.05)$ were entered into the multivariate model. All statistical analyses were performed using SPSS Statistics version 20.0.0 (IBM Corp., Armonk, NY, USA). For all analyses, a two-sided $p$-value $<0.05$ was considered to indicate statistical significance.

\section{Results}

\section{Patients}

The median follow-up period after the first MVAC administration was 10.0 months. The median PFS and overall survival (OS) were 6.5 months (95\% confidence interval [CI] 5.1-7.9) and 14.5 months (95\% CI 7.4-21.4), respectively. The overall response rate was $57.8 \%$. A CR was achieved in two patients (4.4\%) and a PR in twenty-four $(53.3 \%)$. The baseline characteristics of the study population, including the response to first-line GP chemotherapy, are presented in Table 1.

\section{Characteristics of good and poor responses to previous GC chemotherapy}

All included patients were divided into two groups: a good response group and a poor response group, according to the response to first-line GP chemotherapy. The characteristics of the two groups are presented in Table 2. The median treatment-free intervals (TFI) between GP and MVAC chemotherapies for the good and poor response groups were 2.5 and 1.7 months, respectively. There were significant differences in haemoglobin and serum bilirubin levels between the two groups, but not in the variable related to the response to first-line GP chemotherapy (TTP and the number of cycles of first-line GP chemotherapy). The PFS of patients in each of the two groups was calculated using the Kaplan-Meier method. The median PFS after second-line MVAC chemotherapy was 8.0 months (95 \% CI 5.4-10.7) in the good response group and 3.7 months (95\% CI 2.6-4.8) in the poor response group. The results of the log rank test indicated a statistically significant difference $(p=0.008)$ in PFS between the two groups (Fig. 1).

\section{Prognostic predictors of PFS after MVAC chemotherapy}

Univariate and multivariate Cox's regression analyses were performed to identify the independent predictive factors of PFS after MVAC chemotherapy. The results of the univariate and multivariate analyses demonstrated that age (hazard ratio $[\mathrm{HR}]=1.047, p=0.033$ ) and response to first-line GC chemotherapy $(\mathrm{HR}=2.500$, $p=0.012$ ) were predictors of PFS after MVAC chemotherapy (Table 3).
Table 1 Patient characteristics

\begin{tabular}{ll}
\hline Sex & Patients (\%) \\
\hline Female & $0(0.0 \%)$ \\
Male & $45(100 \%)$ \\
Age, median (year, range) & $65(60-72)$ \\
Primary tumor site & \\
Bladder & $30(66.7 \%)$ \\
Ureter or renal pelvic & $6(13.3 \%)$ \\
Ureter or renal pelvic with bladder & $9(20.0 \%)$ \\
Nodal status & \\
N0 & $6(13.3 \%)$ \\
N1 & $8(17.8 \%)$ \\
N2 & $19(42.2 \%)$ \\
N3 & $12(26.7 \%)$
\end{tabular}

Distant metastatic site at the time of MVAC administration

$\begin{array}{ll}\text { Lung } & 21(46.7 \%) \\ \text { Bone } & 16(35.6 \%) \\ \text { Liver } & 9(20.0 \%) \\ \text { Other } & 4(8.9 \%) \\ \text { Absence } & 16(35.6 \%) \\ \text { Single } & 13(28.9 \%) \\ \text { Multiple } & 16(35.6 \%) \\ \text { EOG performance status (PS) } & \\ \text { PS } 0 & 20(44.4 \%) \\ \text { PS } 1 & 23(51.1 \%) \\ \text { PS } 2 & 2(4.4 \%)\end{array}$

MVAC methotrexate vinblastine Adriamycin cisplatin, ECOG Easton Cooperative Oncologic Group

\section{Toxicity}

Anaemia, neutropenia, and thrombocytopenia $\geq$ grade 3 developed in 25,29 , and 20 patients, respectively. A blood transfusion or granulocyte colony-stimulating factor was administered to patients who experienced severe haematological complications. Alopecia was the most common non-haematological toxicity. Most of the nonhaematological toxicities were not life threatening and were tolerated by the patients. The toxicities experienced by the patients are presented in Table 4 .

\section{Discussion}

MVAC elicited a significant response rate in GP-failed patients with advanced urothelial cancer in the current study, which is consistent with the results reported in previous studies using MVAC in the second-line setting. Since Han et al. reported the efficacy and toxicity of MVAC as a second-line chemotherapy, [13] several additional studies have assessed the efficacy of this second-line treatment regimen [10-12]. The current study 
Table 2 Comparison of several factors for patients who were divided by the response to first-line GP chemotherapy

\begin{tabular}{|c|c|c|c|}
\hline & Good $(n=30)$ & Poor $(n=15)$ & $P$ \\
\hline Age, median (year, range) & $64.0(58.5-73.0)$ & $66.0(64.0-71.0)$ & 0.289 \\
\hline Type of platinum compound used as the first-line & & & 0.695 \\
\hline Cisplatin & $23(76.7 \%)$ & $13(86.7 \%)$ & \\
\hline Carboplatin & $7(23.3 \%)$ & $2(13.3 \%)$ & \\
\hline Response to the first-line GP chemotherapy & & & - \\
\hline$C R$ & $11(36.7 \%)$ & - & \\
\hline PR & $19(63.3 \%)$ & - & \\
\hline SD & - & $5(33.3 \%)$ & \\
\hline PD & - & $10(66.6 \%)$ & \\
\hline TTP of the first-line GP chemotherapy, median (months, range) & $11.6(7.8-14.2)$ & $3.13(2.2-5.0)$ & $<0.001$ \\
\hline Cycles of the first-line GP chemotherapy, median (range) & $6.0(6.0-7.3)$ & $4.0(3.0-4.0)$ & $<0.001$ \\
\hline TFI between GP and MVAC, median (months, range) & $2.5(0.7-9.9)$ & $1.7(0.6-3.4)$ & 0.202 \\
\hline Primary tumor site & & & $>0.999$ \\
\hline Bladder & $20(66.7 \%)$ & $10(66.7 \%)$ & \\
\hline Ureter or renal pelvic & $4(13.3 \%)$ & $2(13.3 \%)$ & \\
\hline Ureter or renal pelvic with bladder & $6(20.0 \%)$ & $3(20.0 \%)$ & \\
\hline Clinical N stage & & & 0.453 \\
\hline No & $4(13.3 \%)$ & $2(13.3 \%)$ & \\
\hline N1 & $5(16.7 \%)$ & $3(20.0 \%)$ & \\
\hline N2 & $11(36.7 \%)$ & $8(53.3 \%)$ & \\
\hline N3 & $10(33.3 \%)$ & $2(13.3 \%)$ & \\
\hline \multicolumn{4}{|l|}{ Distant metastatic site at the time of MVAC administration } \\
\hline Lung & $15(50.0 \%)$ & $6(40.0 \%)$ & 0.752 \\
\hline Bone & $10(33.3 \%)$ & $6(40.0 \%)$ & 0.746 \\
\hline Liver & $8(26.7 \%)$ & $1(6.7 \%)$ & 0.234 \\
\hline Others & $4(13.3 \%)$ & - (0.0\%) & 0.285 \\
\hline Hemoglobin (g/dL, range) & $11.3(10.5-11.9)$ & $11.0(9.8-11.3)$ & 0.032 \\
\hline Absolute neutrophil count (/dL, range) & $3751.5(2733.0-5337.0)$ & $3524.0(2415.0-5252.0)$ & 0.596 \\
\hline Platelet count, $10^{3}(/ \mathrm{dL}$, range $)$ & $219.5(184.8-291.5)$ & $216.0(169.0-311.0)$ & 0.952 \\
\hline eGFR (ml/min, range) & $62.6(52.6-75.3)$ & $71.5(47.0-84.7)$ & 0.739 \\
\hline Serum bilirubin (mg/dL, range) & $0.4(0.3-0.6)$ & $0.3(0.3-0.4)$ & 0.047 \\
\hline Serum albumin (g/dL, range) & $4.0(3.8-4.4)$ & $3.8(3.5-4.2)$ & 0.159 \\
\hline ECOG performance status (PS) & & & 0.275 \\
\hline PS 0 & $12(40.0 \%)$ & $8(53.3 \%)$ & \\
\hline PS 1 & $16(53.3 \%)$ & $7(46.7 \%)$ & \\
\hline PS 2 & $2(6.7 \%)$ & - (0.0\%) & \\
\hline
\end{tabular}

GP gemcitabine platinum, $C R$ complete response, $P R$ partial response, $S D$ stable disease, $P D$ progression disease, $T T P$ time to progression, $T F I$ treatment free interval, MVAC methotrexate vinblastine Adriamycin cisplatin, eGFR estimated glomerular filtration rate, ECOG Easton Cooperative Oncologic Group, Boldface significant 2-tailed

demonstrated that MVAC resulted in a significantly higher response rate and longer PFS after the failure of GP in patients with advanced UC who responded to GP as the first-line chemotherapy compared with nonresponders. The response rate and median PFS of patients administered second-line MVAC after GP was $77 \%$ and 8.0 months in responders compared with $40 \%$ and
3.7 months in non-responders, respectively. This suggests that re-challenge using a platinum-based regimen could induce a high response rate and durable PFS after the initial failure of first-line platinum-based chemotherapy in patients with advanced UC. In addition, a higher response rate and longer PFS could be achieved by patient selection. 


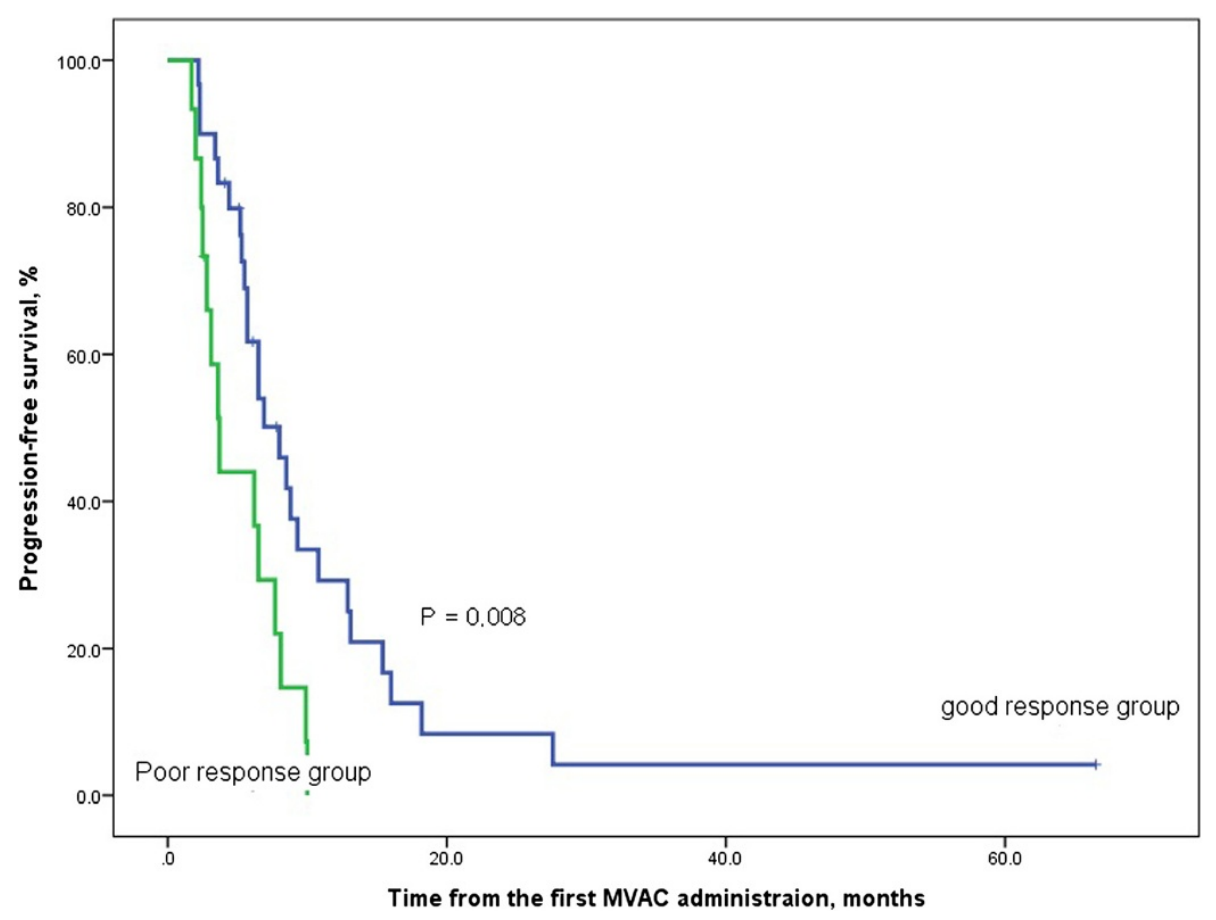

Fig. 1 Kaplan-Meier curve for progression-free survival (\%) in the good response and poor response groups. The good response group included patients who achieved a complete or partial response to first-line GP chemotherapy, whereas the poor response group included individuals with stable or progressive disease after first-line GP chemotherapy

Several studies have reported efforts to find an effective chemotherapeutic regimen after the failure of platinum-based chemotherapy for patients with metastatic, recurrent and inoperable UC [16]. Among the non-platinum-based combination regimens, paclitaxel and gemcitabine have achieved the greatest response rate and PFS of the regimens tested to date in a phase 2 study [17]. Paclitaxel and gemcitabine chemotherapy yielded a $60 \%$ response rate and a 14.4-month median OS. However, 25 of the 41 patients had been treated using first-line platinum-based chemotherapy in a neo-adjuvant or adjuvant setting, and the response rate was only $27 \%$ in the metastatic setting. Bellmunt et al., reported that Vinflunine improved median PFS and OS in a phase 3 study [18]. However, Vinflunine yield only and $8.6 \%$ response rate, and did not demonstrate fully satisfactory survival benefits. Consequently, no satisfactory standard therapy has been found in a second-line setting [19].

Several investigators have questioned whether failure of one platinum-based chemotherapy means true resistance to another platinum-based chemotherapy in UC patients, because different combinations of chemotherapeutic agents exert different synergistic effects on cancer cells, even if they are all based on platinum [10-13, 20]. Edeline et al. and Karadimou et al. suggested that patients with a history of first-line GP chemotherapy in an adjuvant setting and a relatively better ECOG performance status had a more favourable response after second-line MVAC chemotherapy [11, 12]. In addition, Han et al. suggested that patients with a complete or partial response to first-line platinum-based chemotherapy were more likely to show a favourable response to a second-line platinum-based chemotherapy [13]. Gondo et al. also supported a relationship between the responses to first-line and second-line platinum-based chemotherapy in their study on the efficacy and safety of GC as second-line chemotherapy after the failure of firstline MVAC [20]. However, all these studies were limited by a small sample size. The current study is the first to report that the response to first-line GP is a statistically significant predictive marker for response rate and PFS after second-line MVAC, and it also demonstrates that proper patient selection could achieve a higher response rate and longer PFS in patients with advanced UC.

There are several possible explanations for the reresponse phenomenon to platinum-based chemotherapy in patients who failed a first platinum-based chemotherapy. Even after UC cells acquire platinum resistance and the disease progresses, the cells might acquire total resistance to gemcitabine but have incomplete resistance to platinum. Several authors suggested that the synergic effects of combination chemotherapy appear to be able to overcome the resistance of first-line platinum-based chemotherapy in ovarian and lung cancer [21-23]. 
Table 3 Predictors of progression-free survival

\begin{tabular}{|c|c|c|c|}
\hline Variables & $\mathrm{HR}$ & $95 \% \mathrm{Cl}$ & $P$ \\
\hline \multicolumn{4}{|l|}{ Univariate analysis } \\
\hline Age & 1.046 & $1.004-1.089$ & 0.031 \\
\hline Type of platinum compound used as the first-line (cisplatin vs carboplatin) & 1.670 & $0.750-3.718$ & 0.209 \\
\hline TTP of the first-line GP chemotherapy & 0.951 & $0.885-1.022$ & 0.175 \\
\hline Cycles of the first-line GP chemotherapy & 0.947 & $0.834-1.076$ & 0.404 \\
\hline TFI between GP and MVAC & 0.991 & $0.943-1.042$ & 0.735 \\
\hline \multicolumn{4}{|l|}{ Primary tumor site } \\
\hline Bladder vs ureter or renal pelvis/ ureter or renal pelvis with bladder & 1.525 & $0.782-2.975$ & 0.216 \\
\hline Bladder/ ureter or renal pelvis vs ureter or renal pelvis with bladder & 1.735 & $0.806-3.733$ & 0.159 \\
\hline \multicolumn{4}{|l|}{ Nodal status } \\
\hline$N(0$ vs $1,2,3)$ & 1.074 & $0.413-2.789$ & 1.074 \\
\hline $\mathrm{N}(0,1$ vs 2,3$)$ & 0.712 & $0.354-1.431$ & 0.340 \\
\hline $\mathrm{N}(0,1,2$ vs 3$)$ & 0.541 & $0.246-1.193$ & 0.128 \\
\hline \multicolumn{4}{|l|}{ Metastatic site } \\
\hline M0 vs M1 & 1.335 & $0.670-2.658$ & 0.411 \\
\hline Lung & 1.502 & $0.798-2.872$ & 0.207 \\
\hline Bone & 1.207 & $0.631-2.310$ & 0.569 \\
\hline Liver & 1.854 & $0.862-3.987$ & 0.114 \\
\hline Others & 1.056 & $0.371-3.007$ & 0.919 \\
\hline Multi-organ metastasis (absence vs presence) & 1.696 & $0.874-3.294$ & 0.118 \\
\hline Hemoglobin & 0.846 & $0.661-1.082$ & 0.182 \\
\hline Absolute neutrophil count & 1.000 & $1.000-1.000$ & 0.823 \\
\hline Platelet count & 1.001 & $0.997-1.004$ & 1.001 \\
\hline eGFR & 1.000 & $0.979-1.021$ & 0.989 \\
\hline Serum bilirubin & 0.529 & $0.077-3.630$ & 0.517 \\
\hline Serum albumin & 0.417 & $0.161-1.078$ & 0.071 \\
\hline ECOG performance status (0 vs 1,2) & 1.333 & $0.699-2.543$ & 0.382 \\
\hline Response to first-line GP & 2.520 & $1.245-5.015$ & 0.010 \\
\hline \multicolumn{4}{|l|}{ Multivariate } \\
\hline Age & 1.047 & $1.004-1.093$ & 0.033 \\
\hline Response to first-line GP & 2.500 & $1.228-5.098$ & 0.012 \\
\hline
\end{tabular}

$H R$ hazard ratio, $\mathrm{Cl}$ confidence interval, TTP time to progression, GP gemcitabine platinum, TFI treatment free interval, MVAC methotrexate vinblastine Adriamycin cisplatin, eGFR estimated glomerular filtration rate, ECOG Easton Cooperative Oncologic Group, Boldface significant 2-tailed

Combination therapy using more than two agents induces synergistic anti-cancer effects, and different combinations of chemotherapeutic agents can induce different synergies. The MVAC regimen might induce different synergistic effects from those of the GP regimen in UC. Methotrexate, vinblastine and Adriamycin might support the anti-cancer activity of cisplatin differently from that of gemcitabine; therefore, these different effects can affect the response of GP-failed patients to MVAC.

One of the problems with second-line treatment of advanced UC patients is that performance generally deteriorates after the failure of first-line chemotherapy due to cancer progression, delayed recovery of marrow function or decreased organ function. This limits the choice of chemotherapeutic agents in the second-line setting, because the lack of response to second-line treatment can be fatal if it results in further progression of metastases and deterioration of general performance and organ function. Therefore, the appropriate selection of a second-line therapeutic regimen is critical in patients with advanced UC. Additionally, patients receiving MVAC chemotherapy could experience a variety of complications. Several studies have reported MVAC chemotherapy-based toxicities [24-28]. Haematological toxicities developed in $\sim 70 \%$ of the patients in the current study, and many different non- 
Table 4 Toxicities

\begin{tabular}{lll}
\hline Toxicity & All grade $(\%)$ & Grade $\geq 3(\%)$ \\
\hline Hematologic & $34(66.7 \%)$ & $25(49.0 \%)$ \\
Anemia & $40(78.4 \%)$ & $29(56.9 \%)$ \\
Neutropenia & $31(60.8 \%)$ & $20(39.2 \%)$ \\
Thrombocytopenia & & \\
Non-hematologic & $7(13.7 \%)$ & $4(7.8 \%)$ \\
Mucositis & $44(86.3 \%)$ & - \\
Alopecia & $35(68.6 \%)$ & $3(5.9 \%)$ \\
Nausea/vomiting & $28(56.9 \%)$ & $2(3.9 \%)$ \\
Anorexia & $5(9.8 \%)$ & - \\
Diarrhea/constipation & $10(19.6 \%)$ & - \\
Fatigue & $10(19.6 \%)$ & $8(15.7 \%)$ \\
Infection &
\end{tabular}

haematological toxicities also occurred. Because these complications can be life threatening, the use of secondline MVAC chemotherapy should be considered carefully and used only in patients who can benefit from this approach. Therefore, accurately predicting patients who will have a favourable response to second-line MVAC chemotherapy is critical.

The main strength of the current study was the identification of independent predictors of PFS in patients who received second-line MVAC chemotherapy, even though the results might have been affected by the retrospective nature of the study and the small number of patients included in the analysis. These results need to be confirmed and validated by analysing data from a larger prospective study.

\section{Conclusions}

The response to first-line GP is an important predictive and prognostic factor for second-line MVAC in patients with advanced UC. MVAC chemotherapy should be considered as second-line treatment for advanced UC patients who originally responded to GP.

\section{Abbreviations \\ UC: Urothelial carcinoma; GP: Gemcitabine and platinum; GC: Gemcitabine and cisplatin; MVAC: Methotrexate, vinblastine, Adriamycin and cisplatin ECOG PS: Eastern Cooperative Oncology Group performance status; eGFR: Estimated glomerular filtration rate; PFS: Progression-free survival; OS: Overall survival; TTP: Time to progression; TFI: Treatment-free interval; CR: Complete response; PR: Partial response; SD: Stable disease; PD: Progression disease.}

\section{Competing interests}

The authors declare that they have no competing interests.

\section{Authors' contributions}

KS Han was the principal investigator and takes a primary responsibility for the paper; SJ Hong and KS Han provided patient samples and clinical data. KH Kim, SJ Hong and KS Han performed the research; KH Kim and KS Han designed the research; KH Kim and KS Han wrote the paper; SJ Hong and KS Han edited the paper. All authors read and approved the final manuscript.

\section{Acknowledgements}

This work was partly supported by Amanda Hendrix in BioScience. She is a language editor and has made significant revision of the manuscript.

Received: 10 June 2015 Accepted: 16 October 2015

Published online: 27 October 2015

\section{References}

1. Sternberg CN, Yagoda A, Scher HI, Watson RC, Ahmed T, Weiselberg LR, et al. Preliminary results of M-VAC (methotrexate, vinblastine, doxorubicin and cisplatin) for transitional cell carcinoma of the urothelium. J Urol. 1985;133(3):403-7.

2. von der Maase H, Hansen SW, Roberts JT, Dogliotti L, Oliver T, Moore MJ, et al. Gemcitabine and cisplatin versus methotrexate, vinblastine, doxorubicin, and cisplatin in advanced or metastatic bladder cancer: results of a large, randomized, multinational, multicenter, phase III study. J Clin Oncol. 2000;18(17):3068-77.

3. Stenzl A, Cowan NC, De Santis M, Jakse G, Kuczyk MA, Merseburger AS, et al. The updated EAU guidelines on muscle-invasive and metastatic bladder cancer. Eur Urol. 2009:55(4):815-25.

4. Vaughn DJ, Srinivas S, Stadler WM, Pili R, Petrylak D, Sternberg CN, et al. Vinflunine in platinum-pretreated patients with locally advanced or metastatic urothelial carcinoma: results of a large phase 2 study. Cancer. 2009;115(18):4110-7.

5. Dreicer R, Li S, Manola J, Haas NB, Roth BJ, Wilding G. Phase 2 trial of epothilone B analog BMS-247550 (ixabepilone) in advanced carcinoma of the urothelium (E3800): a trial of the Eastern Cooperative Oncology Group. Cancer. 2007;110(4):759-63.

6. Sweeney CJ, Roth BJ, Kabbinavar FF, Vaughn DJ, Arning M, Curiel RE, et al. Phase II study of pemetrexed for second-line treatment of transitional cell cancer of the urothelium. J Clin Oncol. 2006:24(21):3451-7.

7. von der Maase H. Gemcitabine in transitional cell carcinoma of the urothelium. Expert Rev Anticancer Ther. 2003:3(1):11-9.

8. Vaughn DJ, Broome CM, Hussain M, Gutheil JC, Markowitz AB. Phase II trial of weekly paclitaxel in patients with previously treated advanced urothelia cancer. J Clin Oncol. 2002;20(4):937-40.

9. Witte RS, Elson P, Bono B, Knop R, Richardson RR, Dreicer R, et al. Eastern Cooperative Oncology Group phase II trial of ifosfamide in the treatment of previously treated advanced urothelial carcinoma. J Clin Oncol. 1997;15(2):589-93

10. Lee JH, Kang SG, Kim ST, Kang SH, Choi IK, Park YJ, et al. Modified MVAC as a Second-Line Treatment for Patients with Metastatic Urothelial Carcinoma after Failure of Gemcitabine and Cisplatin Treatment. Cancer Res Treat. 2014:46(2):172-7.

11. Edeline J, Loriot Y, Culine S, Massard C, Albiges L, Blesius A, et al. Accelerated MVAC chemotherapy in patients with advanced bladder cancer previously treated with a platinum-gemcitabine regimen. Eur J Cancer 2012:48(8):1141-6.

12. Karadimou A, Lianos E, Pectasides D, Dimopoulos MA, Bamias A. Efficacy of methotrexate/vinblastine/doxorubicin cisplatin combination in gemcitabinepretreated patients with advanced urothelial cancer: a retrospective analysis. Open access journal of urology. 2010;2:193-9.

13. Han KS, Joung JY, Kim TS, Jeong IG, Seo HK, Chung J, et al. Methotrexate, vinblastine, doxorubicin and cisplatin combination regimen as salvage chemotherapy for patients with advanced or metastatic transitional cell carcinoma after failure of gemcitabine and cisplatin chemotherapy. $\mathrm{Br} J$ Cancer. 2008:98(1):86-90.

14. Eisenhauer EA, Therasse P, Bogaerts J, Schwartz LH, Sargent D, Ford R, et al. New response evaluation criteria in solid tumours: revised RECIST guideline (version 1.1). Eur J Cancer. 2009;45(2):228-47.

15. Pond GR, Bellmunt J, Fougeray R, Choueiri TK, Qu AQ, Niegisch G, et al. Impact of response to prior chemotherapy in patients with advanced urothelial carcinoma receiving second-line therapy: implications for trial design. Clin Genitourin Cancer. 2013:11(4):495-500.

16. Sonpavde G, Sternberg CN, Rosenberg JE, Hahn NM, Galsky MD, Vogelzang NJ. Second-line systemic therapy and emerging drugs for metastatic transitional-cell carcinoma of the urothelium. Lancet Oncol. 2010;11(9):861-70.

17. Sternberg CN, Calabro F, Pizzocaro G, Marini L, Schnetzer S, Sella A. Chemotherapy with an every-2-week regimen of gemcitabine and paclitaxel in patients with transitional cell carcinoma who have received prior cisplatin-based therapy. Cancer. 2001;92(12):2993-8. 
18. Bellmunt J, Theodore C, Demkov T, Komyakov B, Sengelov L, Daugaard G, et al. Phase III trial of vinflunine plus best supportive care compared with best supportive care alone after a platinum-containing regimen in patients with advanced transitional cell carcinoma of the urothelial tract. J Clin Oncol. 2009;27(27):4454-61.

19. Network NCC. NCCN clinical practice guidelines in Oncology. Bladder cancer version 2 2014. National Comprehensive Cancer Network, 2014. In.; 2014.

20. Gondo T, Ohori M, Hamada R, Tanaka A, Satake N, Takeuchi H, et al. The efficacy and safety of gemcitabine plus cisplatin regimen for patients with advanced urothelial carcinoma after failure of M-VAC regimen. Int J Clin Oncol. 2011;16(4):345-51.

21. Sekine I, Nokihara H, Horiike A, Yamamoto N, Kunitoh H, Ohe Y, et al. Phase I study of cisplatin analogue nedaplatin (254-S) and paclitaxel in patients with unresectable squamous cell carcinoma. Br J Cancer. 2004;90(6):1125-8

22. Yamada H, Uchida N, Maekawa R, Yoshioka T. Sequence-dependent antitumor efficacy of combination chemotherapy with nedaplatin, a newly developed platinum, and paclitaxel. Cancer Lett. 2001;172(1):17-25.

23. Reed E, Kohn EC, Sarosy G, Dabholkar M, Davis P, Jacob J, et al. Paclitaxel, cisplatin, and cyclophosphamide in human ovarian cancer: molecular rationale and early clinical results. Semin Oncol. 1995;22(3 Suppl 6):90-6.

24. Necchi A, Mariani L, Giannatempo P, Raggi D, Fare E, Nicolai N, et al. Long-term efficacy and safety outcomes of modified (simplified) MVAC (methotrexate/vinblastine/doxorubicin/cisplatin) as frontline therapy for unresectable or metastatic urothelial cancer. Clin Genitourin Cancer. 2014;12(3):203-209.e201

25. Witjes JA, Wullink M, Oosterhof GO, de Mulder P. Toxicity and results of MVAC (methotrexate, vinblastine, adriamycin and cisplatin) chemotherapy in advanced urothelial carcinoma. Eur Urol. 1997:31(4):414-9.

26. Viens P, Gravis G, Bladou F, Lechevallier E, Baume D, Camerlo J, et al. Impact of recombinant human granulocyte colony stimulating factor on dose intensity and toxicity of three cycles of methotrexate, vinblastine, doxorubicin and cisplatin in patients with previously untreated urothelial bladder carcinoma. Eur Cytokine Netw. 1996;7(3):395-9.

27. Igawa M, Kadena H, Ueda M, Usui T. Association between patient characteristics and treatment history, and toxicity associated with methotrexate, vinblastine, adriamycin and cisplatin (M-VAC) for advanced urothelial cancer. Br J Urol. 1994:73(3):263-7.

28. Bahnson RR, Ernstoff MS, Miller RJ, O'Donnell WF. Toxicity comparison of neoadjuvant versus adjuvant methotrexate, vinblastine, doxorubicin, and cisplatin (M-VAC) in radical cystectomy patients. J Surg Oncol. 1990:45(3):143-5.

\section{Submit your next manuscript to BioMed Central and take full advantage of:}

- Convenient online submission

- Thorough peer review

- No space constraints or color figure charges

- Immediate publication on acceptance

- Inclusion in PubMed, CAS, Scopus and Google Scholar

- Research which is freely available for redistribution

Submit your manuscript at www.biomedcentral.com/submit 